\title{
Hipertensão e retinopatia hipertensiva
}

\author{
Hypertension and hypertensive retinopathy
}

\author{
Kenji Sakata ${ }^{1}$ \\ Viviane Sakata $^{2}$ \\ Jackson Barreto Jr. ${ }^{3}$ \\ Kátia M. Bottós ${ }^{4}$ \\ Juliana M. Bottós 5 \\ Newton P. Duarte Filho \\ Daniele Busatto $^{6}$
}

\section{RESUMO}

Objetivo: Avaliar a prevalência de retinopatia hipertensiva ( $\mathrm{RH})$ em pacientes hipertensos (controlados e não controlados) e normotensos na população acima dos 40 anos do município de Piraquara. Correlacionar os casos de alteração retiniana com o sexo, a raça e a idade do paciente. Métodos: Durante 1998 e 2000, foram examinados no Projeto Glaucoma da Universidade Federal do Paraná 1.954 pacientes. Destes, excluíram-se aqueles abaixo de 40 anos e os diabéticos, sendo que a população considerada para este estudo foi de 1.741 pacientes. O protocolo do projeto consiste de anamnese direcionada, aferição da pressão arterial (PA), glicemia por tiras reativas, PIO e fundoscopia direta e indireta. As alterações fundoscópicas pertinentes a retinopatia hipertensiva foram estudadas segundo a classificação de Gans e correlacionadas conforme mencionado anteriormente. Resultados: Dos 1.741 pacientes analisados, $669(38,43 \%)$ são hipertensos, $645(37,05 \%)$ normotensos e $427(24,53 \%)$ suspeitos de hipertensão arterial sistêmica (HAS). Foram encontrados $211(12,12 \%)$ pacientes com sinais de $\mathrm{RH}$, sendo 136 (64,46\%) do sexo femininoe 75 (35,54\%) do sexo masculino; $134(63,98 \%)$ de 40 a 60 anos e $77(36,02 \%)$ com idade superior a 60 anos; predominando as raças branca $(75,83 \%)$ e negra $(11,37 \%)$. Do total de pacientes com RH, 154 (73\%) eram hipertensos, 17 (2,64\%) normotensos e $40(9,37 \%)$ suspeitos de HAS. Dentre os hipertensos com PA controlada, 12,2\% apresentavam sinais de retinopatia. Já dentre os hipertensos com PA não controlada, 25,3\% apresentavam sinais da patologia. Conclusão: A prevalência de RH foi maior nos hipertensos comparativamente aos normotensos e suspeitos de HAS ( $\mathrm{p}<0,001, \mathrm{OR}=5,32$ ). Os pacientes negros $(\mathrm{p}<0,05$ e $\mathrm{OR}=1,67)$, os hipertensos com PA não controlada $(\mathrm{p}<0,01$, $\mathrm{OR}=2,44)$ e os acima de 60 anos $(\mathrm{p}<0,001, \mathrm{OR}=1,85)$ apresentaram maiores chances de desenvolverem RH.

Descritores: Hipertensão/epidemiologia; Pressão arterial; Doenças oculares/diagnóstico; Hipertensão ocular/diagnóstico; Oftalmoscopia

de Oftalmo-Otorrinolaringologia do Hospital das Clínicas da Universidade Federal do Paraná.

${ }^{2}$ Acadêmica do $5^{\circ}$ ano do curso de Medicina da Faculdade Evangélica de Medicina do Paraná.

3 Acadêmico do ano do curso de Medicina da Universidade Federal do Paraná.

${ }^{4}$ Acadêmica do 6ํㅜ período do curso de Medicina da Universidade Federal do Paraná.

${ }^{5}$ Acadêmicos do 7ํㅜ período do curso de Medicina da Universidade Federal do Paraná.

${ }^{6}$ Residente (R2) de Oftalmologia do Hospital das Clínicas da Universidade Federal do Paraná.

Endereço para correspondência: R. Itupava, 1428 . Alto da Rua XV - Curitiba (PR) CEP 80040-000.

E-mail: kenjisakata@hotmail.com

Recebido para publicação em 22.01.2001

Aceito para publicação em 20.08.2001

\section{INTRODUÇ̃̃̃O}

A HAS é a elevação da pressão arterial sangüínea de etiologia multifatorial com repercussões multissistêmicas ${ }^{(1)}$. Entretanto, não existe uma definição rígida quanto ao nível tensional no qual um indivíduo está ou não propenso a desenvolver as complicações pertinentes à doença ${ }^{(2)}$.

As repercussões da HAS se fazem sentir principalmente no leito vascular de órgãos-alvo, entre eles, o olho ${ }^{(1)}$, no qual a retinopatia hipertensiva (RH) se destaca. Várias são as tentativas que visam graduar a $\mathrm{RH}^{(3-4)}$. Classicamente, temos a classificação de Gans, a qual divide as alterações retinianas entre as de etiologia hipertensiva e as ateroscleróticas. Estas são divididas em A0, A1, A2 e A3, as quais representam normalidade, discreta esclerose (alteração do cruzamento arterio-venoso e retificação arteriolar),
\end{abstract}


intensa esclerose (pseudo-esmagamento arterio-venoso e/ou fios de cobre) e obstrução arteriolar (fio de prata), respectivamente. As alterações decorrentes da hipertensão são divididas em H0, H1, H2 e H3, as quais representam normalidade, espasmos arteriolares, espasmos arteriolares associados a hemorragias e exsudatos, e todos estes mais a estase de papila, respectivamente ${ }^{(5-6)}$. Outra classificação também muito difundida é a Classificação Modificada de Scheie que propõe: grau 0 para fundoscopia normal, grau 1 para estreitamentos arteriolares discretos, grau 2 para estreitamentos arteriolares evidentes com irregularidades focais, grau 3 quando associado a hemorragias e/ou exsudatos e grau 4 quando já há edema de disco óptico.

No entanto, a descrição do fundo de olho e os achados angiofluoresceinográficos são mais informativos do que as diversas classificações propostas na literatura ${ }^{(4)}$.

Logicamente, antes de qualquer tentativa de classificar, é necessário reconhecer as lesões. O diagnóstico precoce dos sinais e lesões referentes à RH permite avaliar a gravidade da HAS e, principalmente, realizar um acompanhamento evolutivo das lesões orgânicas hipertensivas, as quais podem freqüentemente levar a escotomas e diminuição da acuidade visual $^{(7)}$. Este acompanhamento, pode ser realizado através de exames oftalmológicos regulares com acuidade visual e oftalmoscopia direta e indireta precisas, juntamente à experiência médico-profissional. É interessante destacar que a fundoscopia é um método prático e fácil para se avaliar os danos em órgãos alvo, além de fornecer informações sobre a atividade e tempo de desenvolvimento da $\mathrm{HAS}^{(8)}$. Estar ciente da utilidade destes exames é mais um meio a ser utilizado pelo clínico para um melhor acompanhamento e tratamento da patologia.

Finalmente, as lesões no fundo de olho, como as hemorragias de retina e microaneurismas, são bastante freqüentes em indivíduos idosos não diabéticos e estão significativamente relacionados com a presença e a severidade da $\mathrm{HAS}^{(9)}$. Baseado nisto, o trabalho em questão tem como objetivos: determinar a prevalência de $\mathrm{RH}$ em hipertensos e normotensos, além de correlacionar a PA controlada ou não, o sexo, raça e a idade com a alteração retiniana.

\section{MÉTODOS}

Foi realizado um estudo prospectivo no Hospital de Clínicas da Universidade Federal do Paraná que se constitui de um programa de "screening" para o Glaucoma, HAS e Diabetes Mellitus (Projeto Glaucoma). Esse estudo foi realizado no período entre 1998 e 2000, sendo que participaram do Projeto: oftalmologistas, residentes em oftalmologia e acadêmicos de medicina.

Foram atendidos 1.954 pacientes pertencentes ao município de Piraquara, cidade da região metropolitana de Curitiba, sendo que destes, 213 foram excluídos. Fatores de exclusão: idade menor que 40 (idade mínima de inclusão no Projeto), ignorância da idade bem como ausência de documentos para comprovação e portadores de DM (a fim de evitar lesões que mascarem a $\mathrm{RH})^{(10-11)}$. Os pacientes atendidos compareceram de maneira voluntária aos Postos de Saúde do município, após adequada divulgação.

Os pacientes foram atendidos segundo um protocolo prédeterminado que consiste de: anamnese, aferição da pressão arterial, aferição da glicemia (com tiras reativas), aferição da pressão intra-ocular pelos tonômetros de Goldmann HaagStreit e fundoscopia realizada 10-20 minutos após a instilação de colírios midriáticos (tropicamida a 1\%) com oftalmoscopia direta e indireta. Quanto à oftalmoscopia direta, utilizou-se do oftalmoscópio direto e, em casos selecionados, a biomicroscopia de fundo.

Todos os pacientes foram submetidos a anamnese com ênfase em queixas oftalmológicas e na história pregressa de hipertensão, diabetes e glaucoma. Realizou-se a dosagem da glicemia utilizando tiras reagentes tipo Dextrostix ${ }^{\circledR}$ e Elite ${ }^{\circledR}$, efetuando-se a leitura no aparelho glucometer e glucometer Elite. A aferição da pressão arterial foi efetuada com o paciente sentado, após 10 minutos de repouso, preferencialmente no braço direito, por acadêmicos de medicina. Quando os níveis pressóricos apresentavam-se iguais ou superiores a 140x90 mmHg, repetia-se a aferição da PA após 5 minutos, permanecendo o paciente em repouso durante este período. Calculava-se a seguir a média entre as duas medidas. $\mathrm{O}$ presente trabalho adotará a definição de que em níveis numéricos, a hipertensão arterial é aquela em que a pressão diastólica média é igual ou superior a $90 \mathrm{mmHg}$, e/ou a pressão sistólica é igual ou maior que $140 \mathrm{mmHg}^{(12)}$ após duas medidas em ocasiões diferentes e/ou uma história de hipertensão com uso de medicamentos anti-hipertensivos no momento do exame. Foram considerados suspeitos de HAS: os pacientes com história ausente de HAS, negativa para o uso de medicação anti-hipertensiva, com PA diastólica igual ou superior a $90 \mathrm{mmHg}$ e/ou PA sistólica igual ou superior a $140 \mathrm{mmHg}$ após duas medidas na mesma ocasião, não sendo possível desta maneira confirmar o diagnóstico de HAS. A hipertensão foi definida como "controlada" caso houvesse história de hipertensão com uso de medicamentos anti-hipertensivos regularmente, pressão diastólica média abaixo de $90 \mathrm{mmHg}$ e a pressão sistólica média menor que $140 \mathrm{mmHg}$. A hipertensão "não controlada" foi definida como história positiva sem uso de medicamentos ou com uso irregular, pressão diastólica igual ou maior que $90 \mathrm{mmHg}$ e/ ou pressão sistólica média maior que $140 \mathrm{mmHg}^{(12)}$.

Os resultados encontrados foram submetidos à estatística descritiva e ao cálculo do quiquadrado corrigido por Yates e do Odds ratio.

Importa ainda ressaltar que os pacientes com alterações na pressão arterial tendo pressão sistólica igual ou acima de $140 \mathrm{mmHg}$, diastólica maior ou igual que $90 \mathrm{mmHg}$ (o que inclui os suspeitos de HAS que são os pacientes nos quais 
não foi possível realizar o diagnóstico definitivo de HAS visto que este é um projeto voltado para o diagnóstico do Glaucoma, sendo somente reavaliados em uma segunda ocasião os pacientes suspeitos para esta patologia) e/ou glicemia superior a $126 \mathrm{mg} / \mathrm{dl}$ em jejum ou $140 \mathrm{mg} / \mathrm{dl}$ pós-prandial foram encaminhados à unidade de saúde ou ao serviço médico que os acompanham.

\section{RESULTADOS}

Foram considerados 1.741 pacientes. Após divisão por sexo: 1.069 pertenciam ao sexo feminino e 672 ao sexo masculino. A distribuição por faixa etária mostrava 1.308 entre 40 e 60 anos e 433 acima de 60 anos. A etnia dividiu-se entre 1.335 brancos, 133 negros, 170 mulatos, 92 mestiços, 3 índios e 8 amarelos.

Do total, 669 (38,43\%) eram hipertensos, 645 (37,05\%) normotensos e $427(24,53 \%)$ suspeitos de hipertensão arterial sistêmica (HAS). No grupo dos hipertensos, 154 (23\%) apresentaram RH, nos normotensos, $17(2,64 \%)$ e nos suspeitos de HAS, $40(9,37 \%)$ pacientes totalizando 211 (12,12\% da amostra total) (Tabela 1).

Dentre os pacientes com RH, 154 (73\%) eram hipertensos, $17(8,06 \%)$ normotensos e $40(18,96 \%)$ suspeitos de HAS. Do total de pacientes com RH, $136(64,46 \%)$ pertenciam ao sexo feminino e $75(35,54 \%)$ ao sexo masculino. A distribuição por faixa etária mostrava que $135(63,98 \%)$ dos pacientes possuíam idades variando de 40 a 60 anos, e $76(36,02 \%)$ com idades superiores a 60 anos. Quanto à etnia, $160(75,83 \%)$ eram brancos, $24(11,37 \%)$ negros, $12(5,68 \%)$ mulatos, $15(7,11 \%)$

Tabela 1. Prevalência da Hipertensão e Retinopatia Hipertensiva na amostra estudada

\begin{tabular}{lc} 
Grupos & Retinopatia Hipertensiva \\
Hipertensos $(n=669)$ & $154(23 \%)$ \\
Normotensos $(n=645)$ & $17(3,64 \%)$ \\
Suspeitos de hipertensão $(n=427)$ & $40(9,37 \%)$ \\
Total $(n=1741)$ & $211(12,12 \%)$ \\
\hline
\end{tabular}

mestiços. Não foram encontrados casos de amarelos ou índios com RH (Tabela 2).

Tomando-se os hipertensos com RH (154 pacientes) procedeu-se a divisão por sexo, faixa etária e raça respectivamente e se verificou que: $45(29 \%)$ pertenciam ao sexo masculino e $109(71 \%)$ ao sexo feminino; 91 (59\%) possuíam idades variando de 40-60 anos e 63 (41\%) acima de 60 anos; finalmente 113 $(73,37 \%)$ dos pacientes pertenciam à raça branca, $21(13,63 \%)$ à negra, $10(6,49 \%)$ à mulata e $10(6,49 \%)$ à mestiça (Tabela 2).

A seguir dividiu-se os normotensos com RH segundo as mesmas variáveis e percebeu-se que dentro dos 17 pacientes normotensos com retinopatia hipertensiva: $10(61,11 \%)$ pertenciam ao sexo masculino e $7(38,88 \%)$ ao sexo feminino; 12 $(72,22 \%)$ possuíam idades entre 40-60 anos e $5(27,78 \%)$ acima de 60 anos; $13(77,78 \%)$ eram brancos, $1(5,55 \%)$ era mulato, 3 $(16,67 \%)$ eram mestiços e $0(0 \%)$ negros. Não foram relatados amarelos ou índios normotensos com retinopatia (Tabela 2).

A divisão dos suspeitos de HAS, 40 no total, segundo as mesmas variáveis obteve os seguintes resultados: $19(47,5 \%)$ pertenciam ao sexo masculino e $21(52,5 \%)$ ao sexo feminino; $29(72,5 \%)$ possuíam idades entre 40 e 60 anos e $11(27,5 \%)$ idade acima de 60 anos; quanto a raça eram $33(82,5 \%)$ brancos, $2(5 \%)$ mulatos, 2 (5\%) mestiços e 3 (7,5\%) negros.

A seguir procedeu-se a divisão em quatro grupos visando a melhor visualização da prevalência da $\mathrm{RH}$ (Gráfico 1). O primeiro grupo contém os hipertensos com PA controlada, somando 115 pacientes (17,2\% dos hipertensos). Estes correspondem àqueles que fazem uso de medicação anti-hipertensiva, acompanhamento médico com a mensuração da pressão arterial regularmente, pressão diastólica média abaixo de 90 mmHg e a pressão sistólica média menor que 140 mmHg. Em 14 $(12,2 \%)$ destes pacientes foi encontrada RH, o que não ocorreu com os 101 pacientes restantes $(87,8 \%)$. O segundo grupo contém os hipertensos com a pressão arterial não controlada: 554 pacientes (82,8\% dos hipertensos). Em 140 (25,3\%) foi encontrada RH e em $414(74,7 \%)$ não foi detectada esta alteração retiniana. $\mathrm{O}$ terceiro grupo corresponde aos normotensos (645 pacientes) onde $17(3,64 \%)$ apresentam RH. O último grupo corresponde aos suspeitos de HAS (427 pacientes) dentre os quais $40(9,37 \%)$ foram diagnosticados com $\mathrm{RH}$.

\begin{tabular}{|c|c|c|c|c|c|c|c|c|c|}
\hline \multicolumn{10}{|c|}{ Tabela 2. Distribuição da amostra com RH } \\
\hline \multicolumn{2}{|c|}{ Distribuição } & \multicolumn{2}{|c|}{ Casos de RH } & \multicolumn{2}{|c|}{$\begin{array}{l}\text { Casos de } \\
\text { hipertensos } \\
\text { com RH }\end{array}$} & \multicolumn{2}{|c|}{$\begin{array}{l}\text { Casos de } \\
\text { normotensos } \\
\text { com RH }\end{array}$} & \multicolumn{2}{|c|}{$\begin{array}{c}\text { Casos de } \\
\text { suspeitos de HAS } \\
\text { com RH }\end{array}$} \\
\hline \multicolumn{2}{|l|}{$\mathrm{N}$} & \multicolumn{2}{|r|}{211} & \multicolumn{2}{|c|}{154} & \multicolumn{2}{|c|}{17} & \multicolumn{2}{|c|}{40} \\
\hline Sexo & $\begin{array}{l}\text { Masc. } \\
\text { Fem. }\end{array}$ & $\begin{array}{r}75 \\
136\end{array}$ & $\begin{array}{l}(35,54 \%) \\
(64,45 \%)\end{array}$ & $\begin{array}{r}45 \\
109\end{array}$ & $\begin{array}{l}(29 \%) \\
(71 \%)\end{array}$ & $\begin{array}{r}10 \\
7\end{array}$ & $\begin{array}{l}(61,11 \%) \\
(38,88 \%)\end{array}$ & $\begin{array}{l}19 \\
21\end{array}$ & $\begin{array}{l}(47,5 \%) \\
(52,5 \%)\end{array}$ \\
\hline \multirow[t]{2}{*}{ Idade } & $40-60$ & 135 & $(63,98 \%)$ & 91 & $(59 \%)$ & 12 & $(72,22 \%)$ & 29 & $(72,5 \%)$ \\
\hline & $>60 a$ & 76 & $(36,02 \%)$ & 63 & $(41 \%)$ & 5 & $(27,78 \%)$ & 11 & $(27,5 \%)$ \\
\hline \multirow[t]{4}{*}{ Etnia } & Brancos & 160 & $(75,83 \%)$ & 113 & $(73,37 \%)$ & 13 & $(77,78 \%)$ & 33 & $(82,5 \%)$ \\
\hline & Negros & 24 & $(11,37 \%)$ & 21 & $(13,63 \%)$ & 0 & $(0 \%)$ & 3 & $(7,5 \%)$ \\
\hline & Mulatos & 12 & $(5,68 \%)$ & 10 & $(6,49 \%)$ & 1 & $(5,55 \%)$ & 2 & $(5 \%)$ \\
\hline & Mestiços & 15 & $(7,11 \%)$ & 10 & $(6,49 \%)$ & 3 & $(16,67 \%)$ & 2 & $(5 \%)$ \\
\hline
\end{tabular}




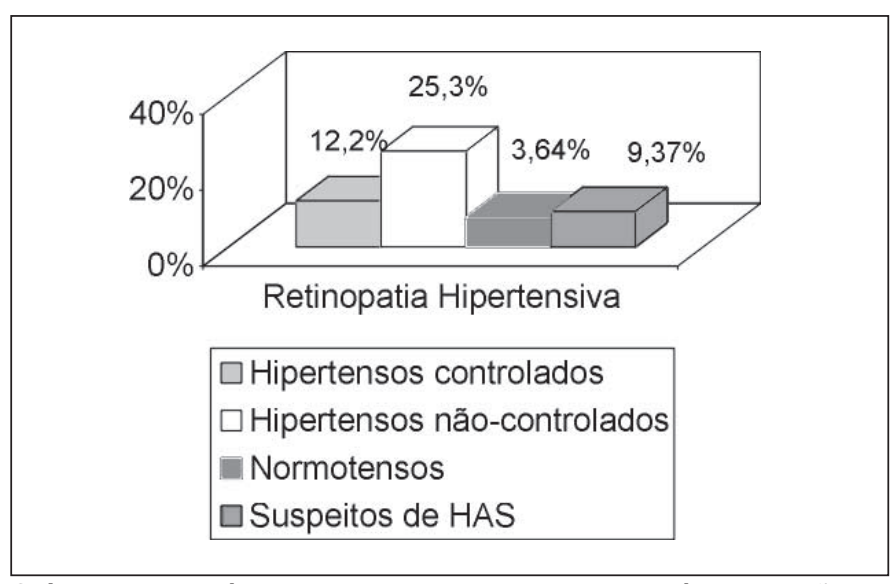

Gráfico 1- Prevalência da RH de acordo com a existência ou não de HAS, bem como o controle desta ou não

\section{DISCUSS ÃO}

A maioria das informações acerca da prevalência das alterações retinianas em hipertensos provém de estudos envolvendo pacientes de clínicas específicas nas quais os indivíduos com patologias severas são encontrados em maior número. O estudo em questão visa avaliar a prevalência da RH na população da região metropolitana de Curitiba (Piraquara) que compareceu voluntariamente para o atendimento. Segundo o censo do IBGE de 1996, o município de Piraquara possui uma população total de 56.057 habitantes, sendo 10.485 com mais de 40 anos. A amostra do presente estudo (1.741 pacientes) representa $16,6 \%$ da população em questão. Deste total de pacientes, os hipertensos representam $38,43 \%$ da população. Este valor está acima do encontrado na literatura ${ }^{(13)}$ na qual os hipertensos representam 30,9\% de uma população com idades variando entre 43 e 86 anos.

Foram encontrados em nosso estudo 211 pacientes de um total de 1.741 pacientes com sinais de RH, representando $12,12 \%$ da população total. Segundo estudo semelhante realizado, 336 pacientes correspondendo a 7,8\% da população total (4.926 pacientes) apresentavam $\mathrm{RH}^{(10)}$.

$\mathrm{Na}$ amostra total, $1.069(61,40 \%)$ pertenciam ao sexo feminino e $672(38,60 \%)$ ao sexo masculino, logo a diferença da prevalência da RH entre os sexos ocorreu pela predominância do $\mathbf{n}$ de mulheres em relação aos homens. Desta maneira, no presente estudo, esta diferença não foi estatisticamente significativa. Na literatura, no entanto, pode-se perceber que a RH foi encontrada predominantemente em mulheres $(R R=1,69) \mathrm{em}$ relação aos homens $(\mathrm{RR}=1,47)^{(13)}$.

Quanto à faixa etária, do total de pacientes, 1.308 (75,13\%) estavam entre 40-60 anos e $433(24,87 \%)$ acima de 60 anos, logo a prevalência de RH foi maior nos pacientes entre 40-60 anos. Contudo, foi a faixa etária acima de 60 anos que demonstrou ser um fator de risco estatisticamente significativo $(\mathrm{p}<0,001, \mathrm{OR}=1,85)$ (Gráfico 2).
É possível ainda realizar análise quanto à distribuição da RH segundo a raça. Pode-se perceber que $75,83 \%$ dos pacientes com RH pertencem à raça branca, fato que se justifica pela predominância desta raça nos pacientes atendidos, correspondendo a 76,68\% da população que compareceu ao atendimento. A raça branca também não demonstrou ser um fator de risco significativo para o desenvolvimento da RH. Os negros, que ocupam o segundo lugar quanto à prevalência de $\mathrm{RH}$ (representando 11,37\% dos pacientes com RH), demonstraram ser um grupo de risco significativo com um $\mathrm{p}<0,05$ (correção de Yates) e um Odds ratio $(\mathrm{OR})=1,67$ (não incluindo a unidade) (Gráfico 2). Cabe aqui destacar que 52,63\% dos negros que compareceram ao estudo são hipertensos, fato que pode explicar a alta incidência da patologia retiniana nesta raça.

Analisando-se ainda a prevalência de retinopatia hipertensiva em pacientes hipertensos, normotensos e suspeitos de HAS pode-se concluir que a pressão arterial é realmente um importante fator de risco, pois $23 \%$ dos hipertensos apresentaram sinais de retinopatia hipertensiva, enquanto somente 2,64\% dos normotensos e 9,37\% dos suspeitos de HAS apresentaram a patologia ( $<<0,001, \mathrm{OR}=5,32)($ Gráfico 2). Os dados encontrados na literatura são semelhantes, segundo os quais: $11 \%$ dos hipertensos apresentam RH em contraste com apenas $6 \%$ dos normotensos ${ }^{(14)}$. Cabe ressaltar também que as anormalidades de fundo de olho (FO) são mais freqüentes em pacientes com pressão diastólica $>105 \mathrm{mmHg}(\mathrm{p}=0,002) \mathrm{e}$ pressão sistólica $>180 \mathrm{mmHg}(\mathrm{p}<0,0001)$ e com duração da hipertensão maior que 3 anos $(\mathrm{p}=0,0002)^{(15)}$. Em pessoas idosas não diabéticas, as lesões retinianas estão significativamente relacionadas à presença e severidade da $\operatorname{HAS}^{(9)}$.

Visando correlacionar a etiologia da retinopatia hipertensiva em pacientes normotensos, após avaliação da pressão arterial durante 24 horas em pacientes normotensos, ou seja, sem pressão arterial maior que 140x90 mmHg pelo esfignomanômetro, e com sinais de retinopatia hipertensiva concluiu-se que estas alterações estavam relacionadas com um quadro hemodinâmico de pré-hipertensão arterial ${ }^{(16)}$. Esta seja talvez uma explicação para a prevalência de RH em normotensos no presente trabalho.

Considerando os $14(25 \%)$ pacientes normotensos com manifestações orgânicas de RH e idade maior que 60 anos, alguns poderiam ser diagnosticados como esclerose involutiva ${ }^{(1)}$.

Não foram encontrados dados na literatura que correlacionassem a ocorrência de PA controlada ou não com o grau de lesão retiniana. Em nosso trabalho, a incidência de RH em paciente sem a PA controlada foi maior que nos pacientes com a PA controlada (25,3\% e 12,2\% respectivamente). A ausência de controle da PA mostrou-se nesse estudo um fator predisponente para o desenvolvimento da $\mathrm{RH}(\mathrm{p}<0,01, \mathrm{OR}=2,44)$ (Gráfico 2). Tal evidência comprova mais uma vez a necessidade de controle da PA visando evitar complicações oculares.

Indubitavelmente, alguns fatores, como os demonstrados no presente estudo, devem ser lembrados para auxiliar no diagnóstico de RH bem como servir de alerta para o médico 


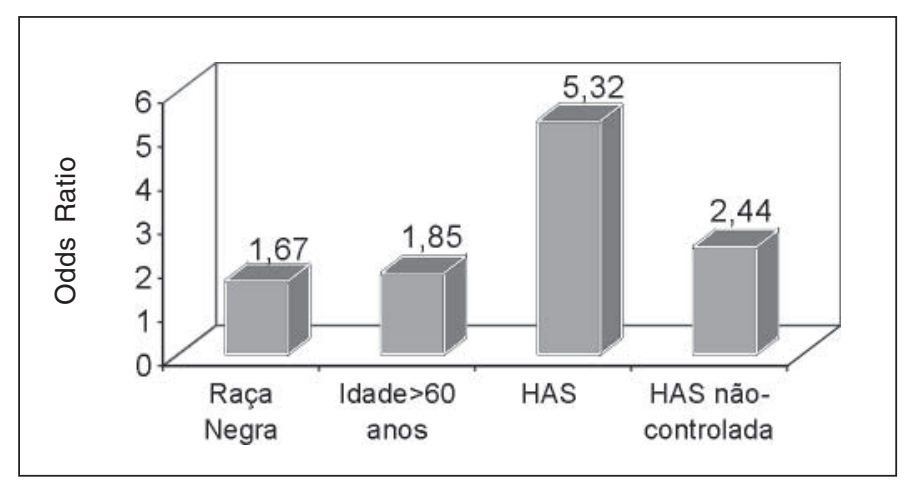

Gráfico 2 - Riscos associados a RH

oftalmologista o qual poderá, por exemplo, estimular ao paciente um melhor controle da PA no caso dos hipertensos, exercendo a medicina preventiva de forma mais efetiva. Mesmo considerando a dificuldade de estagiar a RH através da fundoscopia, comparado a outros métodos como o EEG, raios$\mathrm{X}$ ou dosagem de uréia e creatinina para a avaliação das lesões causadas pela HAS, o exame de fundo de olho mostra-se um meio mais preciso, barato e de fácil execução para triar pacientes com danos nos órgãos-alvo(17).

Portanto, a prevalência de RH foi maior nos pacientes hipertensos comparativamente aos normotensos e suspeitos de HAS. Os pacientes negros, os hipertensos com PA nãocontrolada e os acima de 60 anos apresentam maior risco de desenvolver RH.

\section{ABSTRACT}

Purpose: To investigate the relationship between hypertensive retinopathy (HR) and systemic hypertension (controled or not), gender, age and race. Methods: 1,954 patients who live in Piraquara, aged over 40 years were examined between 1998 and 2000, during a glaucoma, hypertension and diabetes screening program. Diabetics were excluded. Questionnaire, blood pressure, glycemia and IOP measurement, direct and indirect fundoscopy were performed for all 1,741 patients. Gans classification was used to study the retinal changes. Results: Considering 1,741 patients, $669(38.43 \%)$ were hypertensive, 645 (37.05\%) normotensive and 427 (24.53\%) suspected of having hypertension. HR was found in 211 patients $(12.12 \%) .136(64.46 \%)$ were female and $75(35.54 \%)$ male; 135 $(63.98 \%)$ aged between $40-60$ years and $76(36.02 \%)$ aged over 60 years; $75.83 \%$ were white and $11.37 \%$ black; 154 (73\%) were hypertensive, 17 (2.64\%) normotensive and 40 (9.37\%) hyper- tension suspects. $12.2 \%$ and $25.3 \%$ of the hypertensive patients under treatment or not, respectively, had signs of HR. Conclusion: The prevalence of HR was predominant in hypertensive patients comparing to normotensive and hypertension suspects $(\mathrm{p}<0.001, \mathrm{OR}=5.32)$. Hypertensive patients without blood pressure control $(\mathrm{p}<0.01, \mathrm{OR}=2.44)$, aged over 60 years $(\mathrm{p}<0.001, \mathrm{OR}=1.85)$ and/or black ones $(\mathrm{p}<0.05$ e $\mathrm{OR}=$ 1.67) are at increased risk to develop HR.

Keywords: Hypertension/epidemiology; Blood pressure; Retinal diseases/diagnosis; Ocular hypertension/diagnosis; Ophthalmoscopy

\section{REFERÊNCIAS}

1. Matsuhara ML, Brugnara SM. Hipertensão e Retinopatia Hipertensiva. Rev Bras Oftalmol 1995;54:57-9.

2. Schoen FJ. Blood vessels. In: Robbins SL; Cotran RS; Kumar V. Pathologic basis of disease. Philadelphia, Pensylvania: WB Saunders; 1994. p.467-516.

3. Hyman BN. The eye as a target organ: An updated classification of hypertensive retinopathy. J Clin Hypertension 2000;2:194-7.

4. Hayreh SS. Classification of hypertensive fundus changes and their order of appearance. Ophthalmologica 1989;198:247-60.

5. Dodson PM, Lip GYH, Eames SM, Gilson JM, Beevers DG. Hypertensive retinopathy: A review of existing classification systems and a sugestion for a simplified grading system. J Human Hypertension 1996;10:93-8.

6. Hayreh SS. Hypertensive retinopathy. Ophthalmol Clin North Am 1998;11:535-58

7. Williams GH. Doença vascular hipertensiva. In: Harrison TR et al. Medicina interna. México: Interamericana; 1995. p.1170-86.

8. Murua MSE, Chacon TE. Ophthalmoscopic features studied by ophthalmoscopy in arterial hypertension. Rev Mex Oftalmol 1998;72:179-84.

9. Yu T, Mitchell P, Berry G, Li W, Wang JJ. Retinopathy in older persons wihtout diabetes and its relationship with hypertension. Arch Ophthalmol 1998;116:83-9.

10. Klein R, Klein BE, Moss SE, Wang Q. Hypertension and retinopathy, arteriolar narrowing, and arteriovenous nicking in a population. Arch Ophthalmol 1994;112:92-8.

11. Owens DR, Doblen J, Young S, Ryder RE, Jones IR, Vora J et al. Screening for diabetic retinopathy. Diabetic Med 1991;8:S4-10.

12. Abul-Ezz S, Bunke CM, Singh H, Shoh SV. Hipertensão arterial distúrbios vasculares do rim. In: Carpenter A, Plum B. Cecil: Medicina interna básica. Rio de Janeiro: Guanabara Koogan; 1998. p.219-22.

13. Klein R, Klein BE, Moss SE. The relation of systemic hypertension to changes in the retinal vasculature. The Beaver Dam Study Group. Trans Am Ophthalmol Soc 1997;95:329-50.

14. Klein R, Klein BE, Moss SE. Blood pressure, hypertension and retinopathy in a population. Trans Am Ophtalmol Soc 1993;91:207-26.

15. Fuchs FD, Maestri MK, Bredemeir M, Cardozo SE, Moreira FC, Wainstein MV et al. Study of usefulness of optic fundi examination of patients with hypertension in a clinical setting. J Human Hypertension 1995;9:547-51.

16. Cugini P, Cruciani F, Turri M, Regine F, Gherardi F, Petrangeli CM, Gabrieli CB. "Minimal-change hypertensive retinopathy and arterial pre-hypertension", ilustrated via ambulatory blood-pressure monitoring in puntatively normotensive subjects. Int Ophthalmol 1998-99;22:145-9.

17. Hazoff RG, Cunha LAP, Mion DJ, Silva HB, Marcondes M. Previsão da terapêutica em hipertensão arterial - Estudo comparativo do valor da oftalmoscopia e exames complementares. Arq Bras Oftalmol 1987;50:268-73. 